\title{
CÁNCER GÁSTRICO: SOBREEXPRESIÓN DE HER2 POR INMUNOHISTOQUÍMICA HOSPITAL DE SAN JOSÉ, BOGOTÁ DC, 2005 A 2009
}

María del Pilar Archila MD *, Fernando Polo MD**, Gladys Arboleda MD ***, Omar Segura MD, MSc, FETP ****

\section{Resumen}

Introducción: el carcinoma gástrico es una patología cuya incidencia ha aumentado en muchos países, entre ellos Colombia, con alta prevalencia, constituyendo la segunda causa de muerte por cáncer a nivel mundial. Como herramienta que facilita el diagnóstico y el tratamiento, ha surgido en los últimos años la posibilidad de realizar el estudio para determinar la sobreexpresión del gen que codifica el factor de crecimiento epidérmico-2 (HER2) por inmunohistoquímica, en especímenes y biopsias con el fin de dirigir la terapia con trastuzumab a los tumores positivos para esta prueba. Objetivo: determinar la frecuencia de sobreexpresión de HER2 en carcinoma gástrico en nuestra institución, así como obtener datos locales en cuanto a características epidemiológicas. Metodología: se tomaron 58 muestras de tejido de 2005 a 2009 con diagnóstico de adenocarcinoma gástrico en biopsias o gastrectomía en el servicio de patología, a las cuales se les realizó la detección de HER2 por inmunohistoquímica. Resultados: de las 58 muestras 27 fueron biopsias y 31 gastrectomías con predominio de localización en el antro gástrico, de tipo intestinal en su mayoría. Se detectó HER2 positivo en cinco casos, negativo en 51 y dos fueron indeterminados. Conclusión: la frecuencia de sobreexpresión de HER2 en los casos incluidos en el presente estudio es baja, acercándose al límite inferior informado en la literatura.

Palabras clave: cáncer gástrico, adenocarcinoma, sobreexpresión, HER-2, inmunohistoquímica.

\section{GASTRIC CANCER: HER2 OVEREXPRESSION TESTING BY IMMUNOHISTOCHEMISTRY. HOSPITAL DE SAN JOSÉ, 2005 TO 2009}

\begin{abstract}
Introduction: incidence of gastric cancer has increased in many countries including Colombia, showing high prevalence, and remains the second leading cause of cancer mortality in the world. In recent years a new facilitating diagnostic and treatment tool, immunohistochemistry testing to determine the frequency of the gene encoding epidermal growth factor 2 (HER2) overexpression in both surgical and biopsy specimens may be used in order to deliver HER2-positive gastric cancer targeted therapy with trastuzumab. Objective: to determine the frequency of HER2 overexpression in gastric cancer in our hospital, as well as to obtain local data on epidemiologic features. Methodology: from 2005 to 2009, 58 samples obtained by biopsy or gastrectomy were diagnosed as gastric adenocarcinoma at the pathology laboratory. HER2 testing by inmmunohistochemistry was conducted in all 58 samples. Results: out of 58 samples, 27 were biopsies and 31 were gastrectomies predominantly gastric antrum intestinal-type adenocarcinoma. Five cases were HER2 positive, 51 were HER2 negative and 2 were undetermined. Conclusion: frequency of HER2 overexpression of cases included in this study is low, comparable to nearly the lower limit described in literature.
\end{abstract}

Key words: gastric cancer, adenocarcinoma, overexpression, HER-2, immunohistochemistry.

Fecha recibido: junio 27 de 2012 - Fecha aceptado: julio 7 de 2012

Patóloga, Profesora Asociada, Fundación Universitaria de Ciencias de la Salud. Hospital de San José. Bogotá D.C. Colombia.

** Patólogo, Profesor Asistente, Fundación Universitaria de Ciencias de la Salud. Hospital de San José. Bogotá D.C. Colombia.
*** Residente III de Patología, Fundación Universitaria de Ciencias de la Salud, Hospital de San José. Bogotá D.C. Colombia.

**** Epidemiólogo clínico y de campo. Profesor Asistente, Fundación Universitaria de Ciencias de la Salud. Bogotá D.C. Colombia. 


\section{Introducción}

El cáncer gástrico en el mundo se ha convertido en una enfermedad cada vez más frecuente con un alto número de muertes, a pesar de los esfuerzos por realizar un diagnóstico temprano y un tratamiento oportuno. En la actualidad es el segundo carcinoma más frecuente y presenta alta incidencia, sobre todo en países de Asia y Suramérica. ${ }^{1}$ En Colombia es el segundo tumor maligno más común en ambos géneros y es la primera causa de muerte por cáncer. ${ }^{2,3}$

El diagnóstico se realiza mediante endoscopia de vías digestivas altas y biopsia. Con frecuencia se encuentra en un estadío avanzado en ese momento. Su pronóstico depende de la profundidad de la invasión tumoral, el tipo histológico y la extensión del tumor, y el pilar del tratamiento es la gastrectomía acompañada de terapias adyuvantes como radio y quimioterapia. ${ }^{4.5}$

En el camino para encontrar herramientas con el fin de optimizar el manejo del carcinoma gástrico, se ha enfocado el estudio en la categorización molecular de la enfermedad ${ }^{6,7}$ y los avances más importantes se han dado en las pruebas para detectar la sobreexpresión del receptor del factor de crecimiento epidérmico humano tipo 2 (HER2), ya sea por inmunohistoquímica o por estudios genéticos. ${ }^{8}{ }^{89}$ Dicha sobreexpresión le confiere al tumor un mayor número de receptores en sus células para factor de crecimiento y por lo tanto una alta tasa de división y proliferación. De esta manera, se ha visto que los tumores que expresan un mayor número de copias de HER-2 tienen un peor pronóstico y menor respuesta al tratamiento convencional. ${ }^{10,11}$

El HER2 es un receptor codificado por el gen erbB2, que se encuentra en el brazo largo del cromosoma 17 y forma parte de la familia de receptores tirosina quinasa, constituido por un dominio intracelular, uno transmembrana y uno extracelular. Para la activación del receptor es necesario que se efectúe una unión al ligando con dimerización y posterior fosforilación en el dominio intracelular, con lo que se activan factores promotores de la actividad mitótica y antiapoptóticos. En condiciones normales cada célula tiene dos copias del gen erbB-2, pero en algunos tumores se ha observado que sus células poseen múltiples copias de este gen (amplificación genética), lo que genera una mayor cantidad de receptores en la superficie celular que se dimerizan sin necesidad de unirse al ligando, por lo que la célula continuará respondiendo en forma descontrolada al estímulo del receptor, proliferando sin inhibición.

Para la detección de la proteína producto de la transcripción del gen erbB-2 se ha utilizado la técnica de inmunohistoquímica, que se basa en el principio de unión de un antígeno a un anticuerpo marcado con un cromógeno para hacerlo visible al microscopio de luz. Otro método se basa en la cuantificación directa del número de copias del gen en el núcleo de la célula tumoral, con la técnica de fluorescencia por hibridización in situ (FISH por sus siglas en inglés), siendo más precisa y confiable, pero más costosa.

En este ámbito se ha avanzado mucho en cáncer de seno, en el cual se realiza de rutina la detección de HER2 desde hace varios años, con el fin de dar manejo específico con trastuzumab. Este anticuerpo monoclonal actúa uniéndose al dominio extracelular del HER2, compitiendo con los factores de crecimiento y evitando su unión al ligando para bloquear la activación de la cascada y la proliferación celular. ${ }^{12}$ La molécula de trastuzumab se aprobó para el tratamiento del cáncer de seno hace más de diez años y en el cáncer gástrico hace cerca de un año. ${ }^{13,14}$

Se han realizado estudios con este fármaco en adenocarcinoma gástrico demostrando un aumento de la sobrevida hasta de seis meses en pacientes con estadíos avanzados de la enfermedad, cuando se administra a pacientes con tumores positivos para HER2, en combinación con otros agentes quimioterapéuticos. ${ }^{10,15,16}$ Por lo tanto, ya ha sido implementado en numerosos sitios como manejo de primera línea en los tumores que sobreexpresan el receptor. ${ }^{17,18}$

En el país aún no existen datos acerca de esta sobreexpresión, por lo que el objetivo del presente estudio es determinar su frecuencia en carcinoma gástrico en nuestra institución, así como obtener datos locales en cuanto a características epidemiológicas de dicha patología. 


\section{Metodología}

Estudio observacional de corte transversal en el que se tomaron los bloques de parafina de tejidos de pacientes con diagnóstico de adenocarcinoma gástrico en el archivo de patología del Hospital de San José de Bogotá DC entre los años 2005 y 2009, que estuvieran en adecuado estado de conservación. De los pacientes en los que se encontraron tanto biopsia como gastrectomía, se utilizó el tejido que tuviera mejor calidad.

Se tomaron datos del registro del servicio de patología, así como de las historias clínicas disponibles en el hospital para diligenciar los formatos de recolección incluyendo variables como género, edad, tipo de muestra, tipo histológico, localización y TNM. Se revisaron las láminas de hematoxilina-eosina con un patólogo experto del Hospital de San José para estudiar las de mejor calidad y mayor cantidad de material. Se escogió una lámina de cada caso con su respectivo bloque de parafina en el cual se realizó el estudio manual de HER2 por inmunohistoquímica utilizando el kit HercepTest TM, DAKO Denmark A/S, de acuerdo con la especificación dada por el fabricante y los protocolos del servicio.

Los resultados fueron analizados e interpretados por un patólogo con entrenamiento en inmunohistoquímica y clasificados según consenso internacional expuesto en la Tabla 1. ${ }^{19,20}$ Los interpretados como indeterminados fueron leídos por un segundo patólogo.

La recopilación de los resultados se hizo en el software Microsoft Excel@ 2007 y el análisis de los datos se realizó con el programa estadístico Stata 10 con el cual se realizó agrupación de variables en frecuencias absolutas y relativas, y el análisis de medidas de tendencia (central, dispersión). El estudio fue aprobado por el comité de investigaciones y ética de la Facultad de Medicina de la Fundación Universitaria de Ciencias de la Salud, el cual fue considerado de riesgo bajo.

\section{Resultados}

Se encontraron en total 64 casos con diagnóstico de adenocarcinoma gástrico en los archivos de patología

\begin{tabular}{|c|c|}
\hline Caracteristicas de reactividad & $\begin{array}{l}\text { Puntuación/ } \\
\text { clasificación }\end{array}$ \\
\hline $\begin{array}{l}\text { No reactiva o reactividad de mem- } \\
\text { brana en menos del } 10 \% \text { de las cé- } \\
\text { lulas tumorales. }\end{array}$ & 0/negativo \\
\hline $\begin{array}{l}\text { Reactividad de membrana débil o } \\
\text { incompleta en más del } 10 \% \text { de las } \\
\text { células tumorales (Figura } 5 \text { ). }\end{array}$ & I+/negativo \\
\hline $\begin{array}{l}\text { Reactividad moderada en la membra- } \\
\text { na basal o completa, en más del } 10 \% \\
\text { de las células tumorales (Figura } 6 \text { ). }\end{array}$ & $2+$ /indeterminado \\
\hline $\begin{array}{l}\text { Reactividad fuerte en la membrana } \\
\text { basal o completa, en más del } 10 \% \\
\text { de las células tumorales (Figura } 7 \text { ). }\end{array}$ & 3+/positivo \\
\hline
\end{tabular}

* En biopsias se considera positiva la marcación fuerte en la membrana basal o completa en un grupo de al menos cinco células, independiente del porcentaje de la muestra.

del Hospital de San José entre 2005 y 2009, de los cuales fueron excluidos dos por ausencia del bloque de parafina en el archivo y en dos casos se encontraron muestras correspondientes a biopsia y gastrectomía del mismo paciente, por lo cual se escogió el material con mejor preservación en cuanto a fijación y conservación. Se realizó el estudio de HER2 por inmunohistoquímica en los 60 bloques de parafina, en dos de ellos no se encontró tumor en los nuevos niveles de corte por desgaste del tejido, por lo que se obtuvieron en total 58 casos para análisis. De estos, 27 (46.5\%) fueron biopsias y $31(53.4 \%)$ gastrectomías, la edad promedio de presentación fue 61 años, con mínimo de 34 y máximo de 88 (DE: 13.7). 27 (46.5\%) correspondieron a mujeres. Para determinar el tipo histológico se utilizó la clasificación de Lauren, con un predominio del adenocarcinoma de tipo intestinal en 41 casos $(70.7 \%)$ y difuso en 17 (29.3\%). Así mismo, se encontró que 31 casos $(53.4 \%)$ se localizaron en el antro, $13(22.4 \%)$ en el cuerpo, cinco (8.6\%) en el cardias, tres $(5.1 \%)$ fueron pilóricos y uno $(1.7 \%)$ en el fondo; en cinco no se especificó la localización de la toma.

Se realizó la prueba de HER2 por inmunohistoquímica en los 58 bloques de parafina encontrando cinco casos positivos $3+(8.6 \%)$, dos casos indeterminados 
$2+(3.4 \%)$ y 51 negativos $(87.9 \%)$, de los cuales 45 se clasificaron como 0 y seis con $1+$ (Figura 1). Como datos relevantes, en el cruce de variables se encontró un claro predominio de los casos positivos para HER2 en el adenocarcinoma de tipo intestinal, de tal manera que el $100 \%$ de las muestras que mostraron sobreexpresión por inmunohistoquímica fueron de este tipo histológico (Figura 2).

En la comparación por género hubo un mayor número de sobreexpresión en mujeres, con cuatro casos

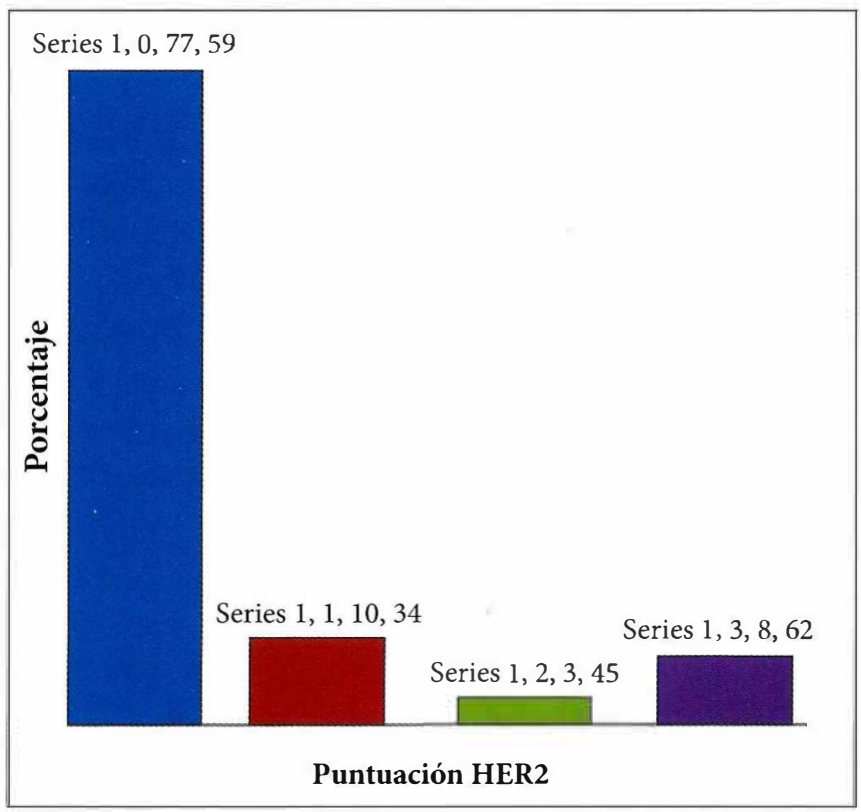

Figura I. Frecuencia de sobreexpresión de HER2.

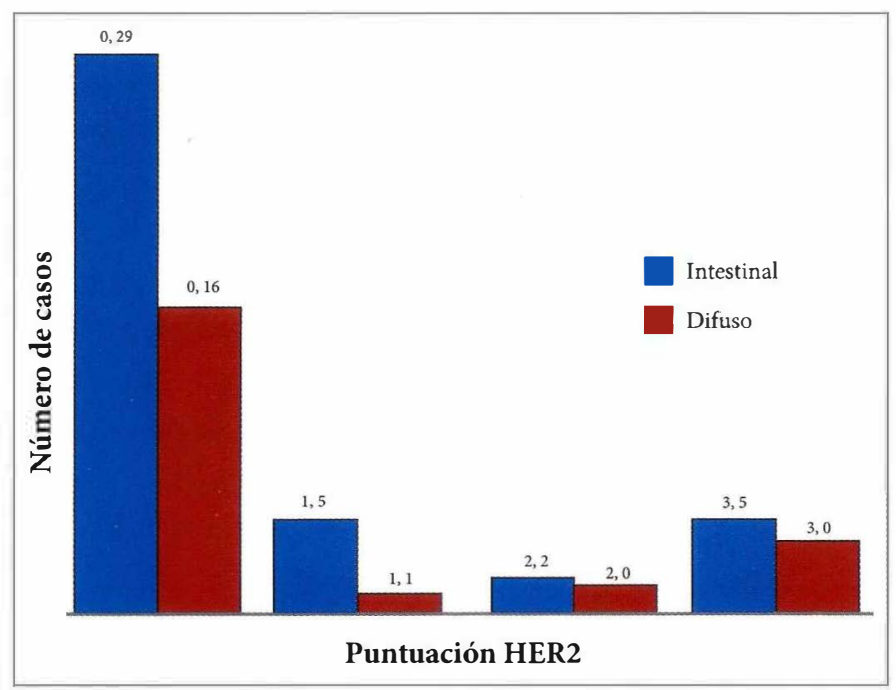

Figura 2. Sobreexpresión de HER2 por tipo histológico.
HER2 positivo de $27(14.8 \%)$ contra un caso HER2 positivo de 31 (3.2\%) en hombres (Figura 3). Se agruparon por grupos de edad en mayores y menores de 50 años, encontrando tres HER2 positivos de 15 (20\%) en menores de 50 años y sólo dos HER2 positivos de 43(4.6\%) en mayores de 50 (Figura 4). Se trató de establecer el estadio tumoral por TNM en las historias clínicas, pero el seguimiento de la mayoría de los casos no se realizó en el hospital, por lo que la información fue insuficiente y se excluyó del análisis final de los datos.

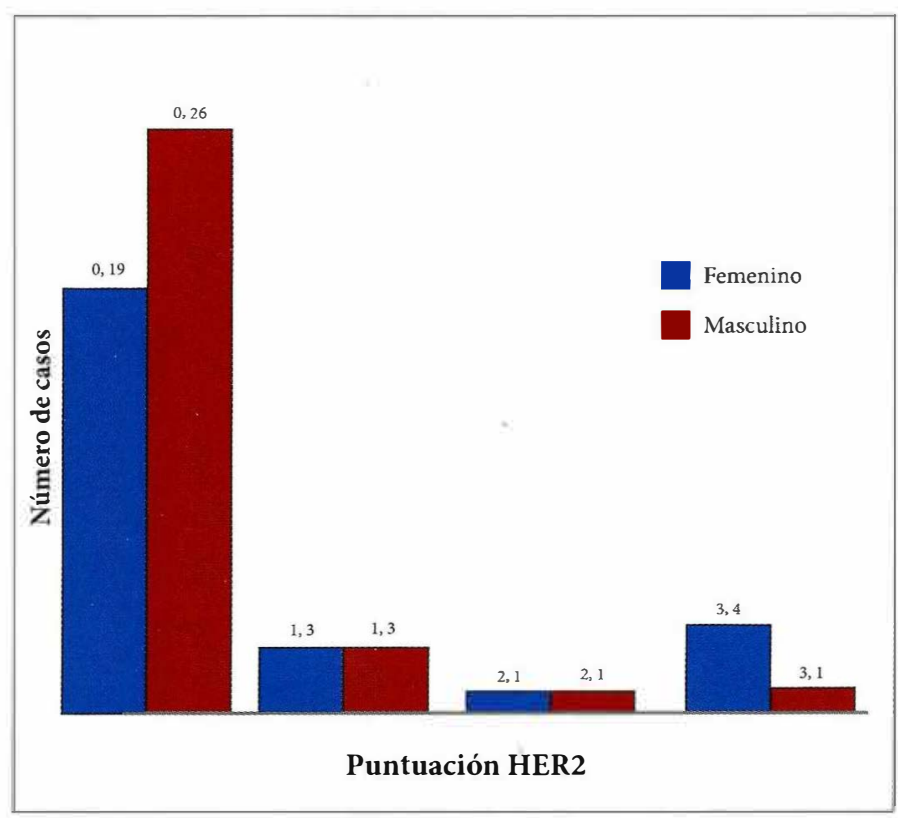

Figura 3. Sobreexpresión de HER2 según género.

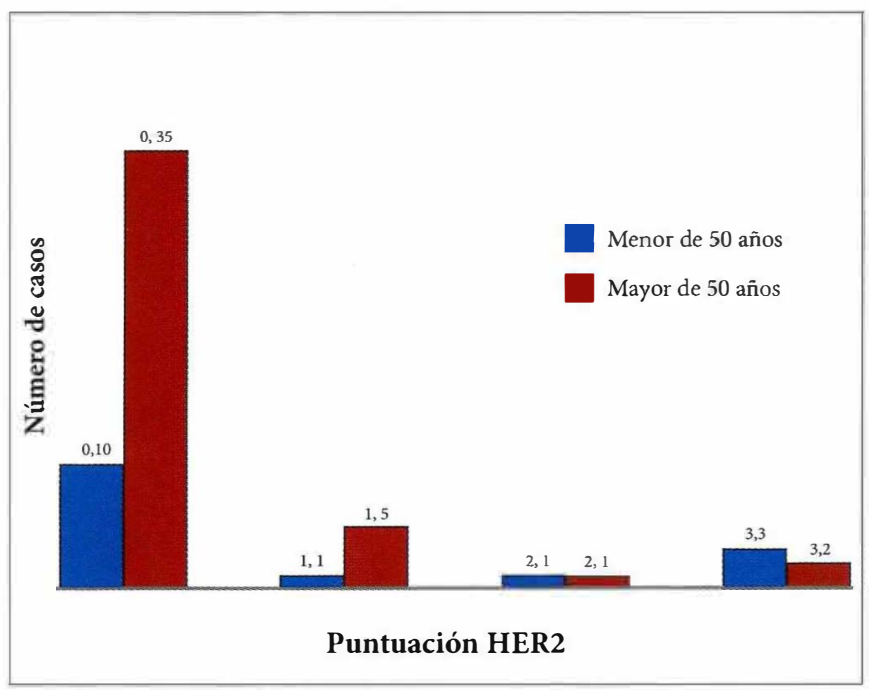

Figura 4. Sobreexpresión de HER2 en grupos de edad. 


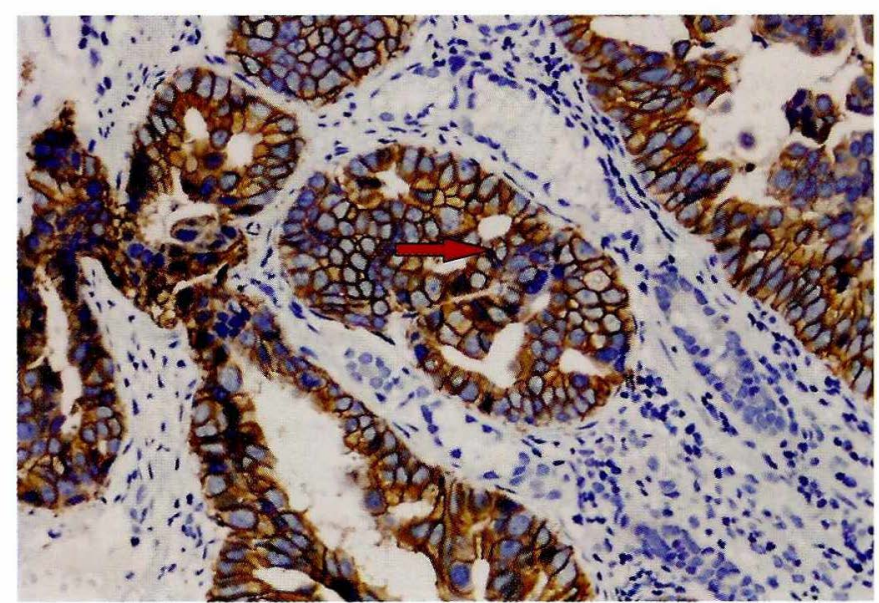

Figura 5. HER2 positivo $3+$ por inmunohistoquímica (40x). Tinción fuerte en membrana citoplasmática basolateral (flecha) en más del 10\% de las células.

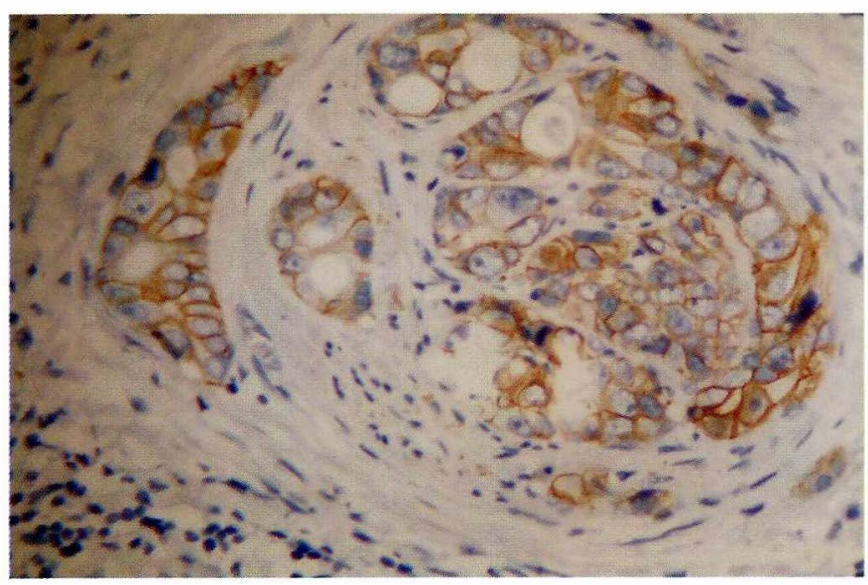

Figura 6. HER2 indeterminado 2+ por inmunohistoquímica (40x). Tinción moderada e incompleta de la membrana citoplasmática en más del $10 \%$ de las células tumorales.

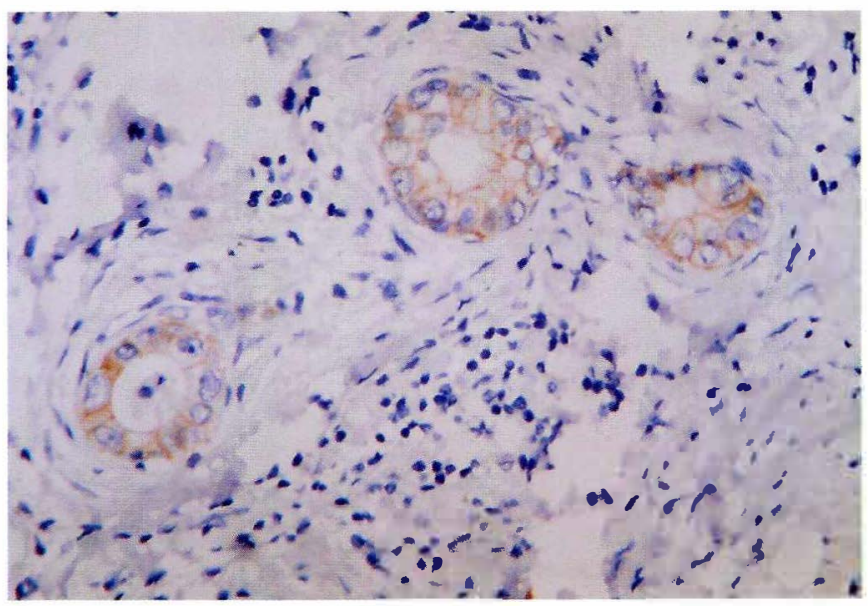

Figura 7. HER2 negativo I+ por inmunohistoquímica(40x). Tinción débil e incompleta de la membrana citoplasmática en más del $10 \%$ de las células tumorales.

\section{Discusión}

En este estudio se buscó determinar la frecuencia de la sobreexpresión de HER2 y caracterizar otras variables que permitan acercarnos al comportamiento epidemiológico del adenocarcinoma gástrico en nuestro medio. En los casos que cumplieron los criterios para ingresar, se halló como principal resultado la sobreexpresión de HER2 en cinco de los 58 casos, lo cual corresponde a un $8.6 \%$, que está en el rango de los datos reportados en la literatura mundial, en la cual se ha visto una positividad por inmunohistoquímica desde $8.2 \%$ hasta $53.4 \%$ en diferentes países del mundo. ${ }^{10,18}$

En cuanto a otras variables evaluadas, se encontró la edad promedio en 61 años y predominio en la población mayor de 50 años (43 casos de 58), lo cual está acorde con lo reportado para este tipo de patología. ${ }^{15}$ Sin embargo, hubo tres pacientes menores de 40 años, lo que lleva pensar que la edad de presentación ha disminuido y es importante tener en cuenta esta variación a la hora del tamizaje. Observamos que el número de hombres y mujeres es muy cercano, con sólo un ligero predominio de los primeros ( 31 de 58), lo cual difiere con la literatura previa en la que hay amplia mayoría del género masculino., 30

Acorde a lo encontrado en la literatura, el mayor número de casos fue localizado en el antro (53.4\%) y el tipo histológico más frecuente fue el intestinal (70.6\%). En el cruce de variables se encontraron algunas asociaciones importantes, aunque sin significancia estadística por el bajo número de la muestra. Se observó mayor sobreexpresión de HER2 en menores de 50 años, así como predominio de los casos positivos en mujeres y en los adenocarcinomas de tipo intestinal. ${ }^{20}$

Dada la importancia que ha tomado la sobreexpresión del HER2 en el seguimiento y tratamiento del carcinoma gástrico y la gran variación que hay entre las diferentes publicaciones alrededor del mundo, es relevante reportar los datos encontrados en este estudio para aproximarnos al comportamiento de esta enfermedad en nuestra institución y saber que esperar a la hora de evaluar estos pacientes en un futuro. Así mismo, teniendo en cuenta las variaciones de la técnica y la interpretación con respecto a la misma realizada en el 